\title{
Wake Characteristics of Vane-Type Vortex Generator
}

\author{
HoJoon Shim ${ }^{1}$, JongSung Lee ${ }^{2}$, Hoon Chae ${ }^{3}$, Seung-O Park ${ }^{4}$ \\ ${ }^{1,2,3}$ Hanwha Corporation Defense R\&D Center \\ 99 Oesam-ro 8 Beon-gil Yuseong-gu, Daejeon, Republic of Korea \\ ${ }^{4}$ Korea Advanced Institute of Science and Technology \\ 291 Daehak-ro Yuseong-gu, Daejeon, Republic of Korea \\ hjshim2461@gmail.com; sopark@kaist.ac.kr
}

\begin{abstract}
Vortex generator is commonly used to control flows and delay separation in many engineering applications because of its geometrical simplicity and high performance improvement. Studies on the wake characteristics concerning development, interaction, and decay of various streamwise vortices depending on the generator shape at a same test condition are seen to be somewhat rare. In this regard, the present study was carried out. To examine the effect of the vortex generator shape, wake characteristics of a triangular, a trapezoidal, and a rectangular vane-type vortex generator were investigated for both cases of the flat plate laminar and turbulent boundary layer. The height of the generator was chosen to be about the boundary layer thickness at the position of its installation for both conditions. Two different lengths of the generator were chosen: two and five times the height. The laminar and turbulent boundary layer thicknesses at the vortex generator installation location were approximately $5 \mathrm{~mm}$ and $15 \mathrm{~mm}$, respectively. Stereo-PIV measurements in cross flow planes downstream of the three vane-type vortex generators were conducted. Wake characteristics such as overall vortical structure and vorticity distribution at several downstream distances were obtained from the PIV data. Wake characteristics, as expected, were found to vary strongly with the geometry and angle of attack so that no general tendency could be deduced. In the case of the laminar flow case, two streamwise vortices were observed at specific test conditions. However, a single streamwise vortex was only generated for the case of the turbulent flow.
\end{abstract}

Keywords: Vortex Generator, Boundary Layer, Flat Plate, Stereoscopic PIV

\section{Introduction}

As is well known, vortex generator is a typical passive flow control device and is used to delay flow separation in many engineering applications. A study about vortex generator was first carried out by Taylor [1]. He found that streamwise vortices generated by the small vane vortex generators increase the near-wall momentum through momentum transfer from outer flow to near wall region.

The height of the vortex generator is an important design parameter. The shape of the vortex generator is obviously an important factor as well. There are various vortex generator shapes; triangular, trapezoidal, rectangular, wishbone and doublet, etc. Torii and Yanagihara investigated the influence of a triangular vortex generator on heat transfer in laminar boundary layer [2]. Yanagihara and Torii also investigated the influence of vortex generator shape (triangular and rectangular) on heat transfer in laminar boundary layer [3]. They found that the rectangular vortex generator with higher angle of attack or larger frontal area showed the best heat transfer performance. Ashill et al. carried out experiments on wedge and triangular vane types of vortex generators of $h / \delta=0.3$ on a bump [4]. Yao et al. measured flow field downstream of a single rectangular vortex generator using stereoscopic particle image velocimetry (Stereo-PIV herein after) [5]. Angele and Muhammad-Klingmann performed experiments over a separating boundary layer using rectangular vortex generator with three different heights and streamwise positions [6]. Lin et al. conducted experimental study to evaluate boundary layer separation control using triangular and trapezoidal shapes of small surface-mounted vortex generators on a high lift airfoil with a leading edge slat and a flap [7]. Godard and Stanislas carried out parametric study (angle of attack, length, transverse distance, etc.) of vortex generators (triangular and rectangular) and tested both corotating and counter-rotating configurations [8]. They found that triangular vortex generators produced a significant drag improvement compared to rectangular vortex generators. The representative shapes of the vane-type vortex generator were triangular and rectangular. Most of experimental and computational studies were mainly conducted in turbulent boundary 
layer. Kerho et al. conducted experimental study to examine the effect of wishbone and ramped cone generators on airfoil at low Reynolds number condition [9]. They found that the wishbone vortex generators and ramped cones delayed the onset of the separation bubble. Velte et al. carried out the wake measurements in a wind tunnel and the flow visualization in a water tunnel past the rectangular vortex generator in laminar boundary layer [10]. They found that the vortical structure in the wake downstream of the vortex generator was much more complicated than a simple tip vortex structure. Yanagihara and Torii showed that the longitudinal vortices around the triangular vortex generator were composed of the main vortex, the corner vortices, and the induced secondary vortex [11]. In the laminar boundary layer, Velte et al. [10] and Yanagihara and Torii [11] showed that various vortices were generated by a vortex generator.

The present study focuses on the development of streamwise vortices of a vortex generator over a boundary layer. Wake characteristics of a triangular, a trapezoidal, and a rectangular vortex generator were investigated for the laminar and the turbulent boundary layer conditions. Stereo-PIV measurements in cross flow planes downstream of the three vane-type vortex generators were conducted.

\section{Experimental Setup and Numerical Simulation}

\subsection{Test Facility and Flat Plate}

This study was carried out in the subsonic wind tunnel of the Korea Aerospace Research Institute (KARI). The wind tunnel has a test section of $1.0 \mathrm{~m}$ width, $0.75 \mathrm{~m}$ height and $2.0 \mathrm{~m}$ length. The wind speed ranges from $1 \mathrm{~m} / \mathrm{s}$ to $110 \mathrm{~m} / \mathrm{s}$ [12]. This study was carried out at the wind speed of $10 \mathrm{~m} / \mathrm{s}$. The streamwise turbulence intensity was $0.06 \%$.

The flat plate for vortex generator installation was made of aluminium and the supports were installed to adjust the height and the horizontality of the flat plate. The flat plate was $990 \mathrm{~mm}$ wide, $1866 \mathrm{long}$, and $12 \mathrm{~mm}$ thick as sketched in Fig. 1 where the coordinate system adopted in this study is also shown. The $\mathrm{z}$ axis is along the center line of the flat plate and the $y$ axis is normal to the upper surface of the flat plate. The flat plate was mounted at the center plane of the wind tunnel test section. The flat plate surface was covered with the black thin film to reflect the laser in one direction but not to allow the reflected laser into the camera. The leading edge of the flat plate was of a super-ellipse shape to prevent the leading edge separation [13]. The velocity profile on the flat plate was first measured by using 2-dimensional particle image velocimetry (2D-PIV). The velocity profile at $750 \mathrm{~mm}$ from the leading edge was measured. The velocity profile without the trip strip was found to be that of the Blasius profile, and the flat plate boundary layer was tripped to generate the turbulent boundary layer.

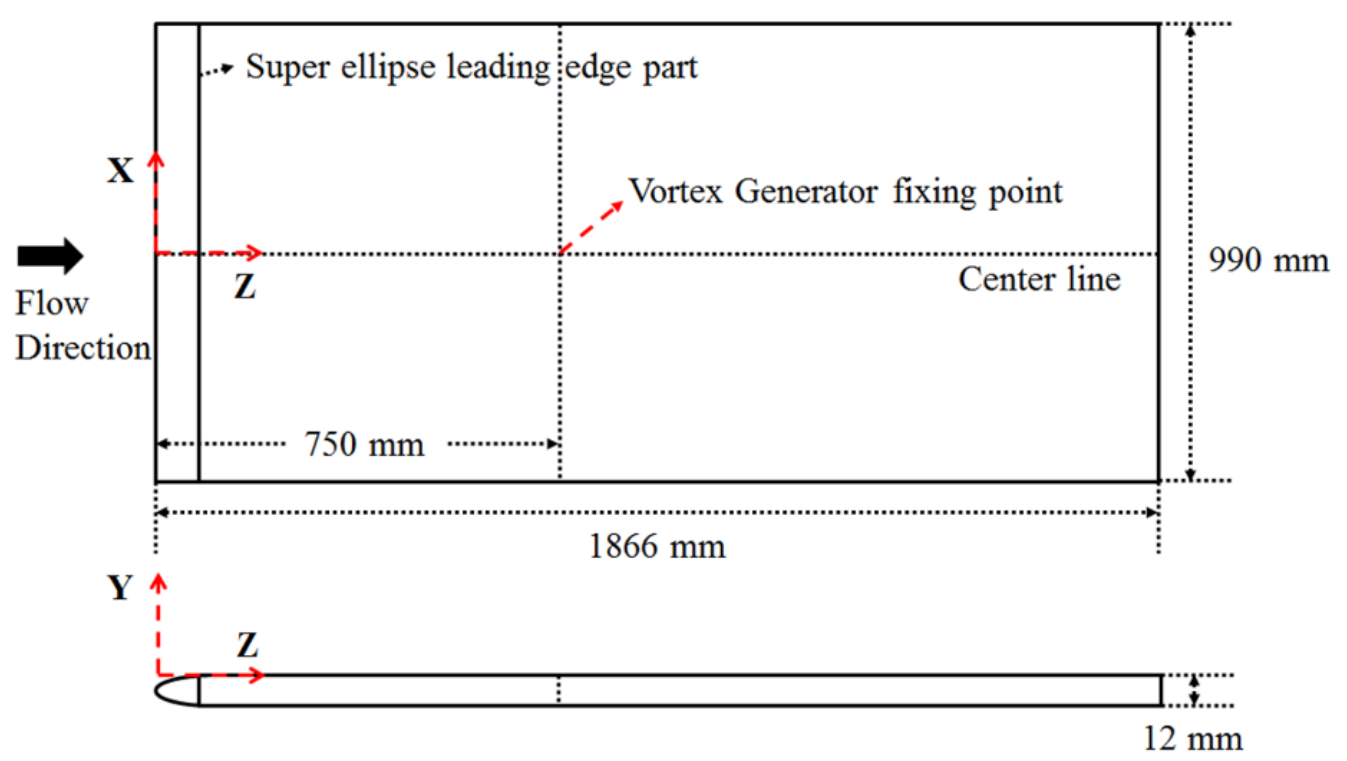

Fig. 1: Schematic of geometry and coordinate system of flat plate with super ellipse leading edge. 


\subsection{Vortex Generator}

In this study, three different planform shapes of vortex generator were considered: triangular, trapezoidal, and rectangular shapes as shown in Fig. 2 where test conditions are also given. The heights of the vortex generator were set to be the same as the boundary layer thickness at the installation location of $750 \mathrm{~mm}$ (see Fig. 1). The laminar boundary layer thickness, $\delta_{\mathrm{l}}$, at the fixing point position was approximately $5 \mathrm{~mm}$, and the turbulent boundary layer thickness, $\delta_{\mathrm{t}}$, was approximately $15 \mathrm{~mm}$. Godard and Stanislas recommended that a minimum value of the generator length was two times the height [8]. Thus, one length of the two times height was selected. To look into the effect of length, a much longer length of five times the height was also chosen. To investigate the influence of the angle of attack, three angles of attack $\left(10^{\circ}, 15^{\circ}\right.$, and $\left.20^{\circ}\right)$ were selected. To generate angle of attack, the vortex generator was rotated about the fixing point positioned at $2.5 \mathrm{~mm}$ ahead of the trailing edge.

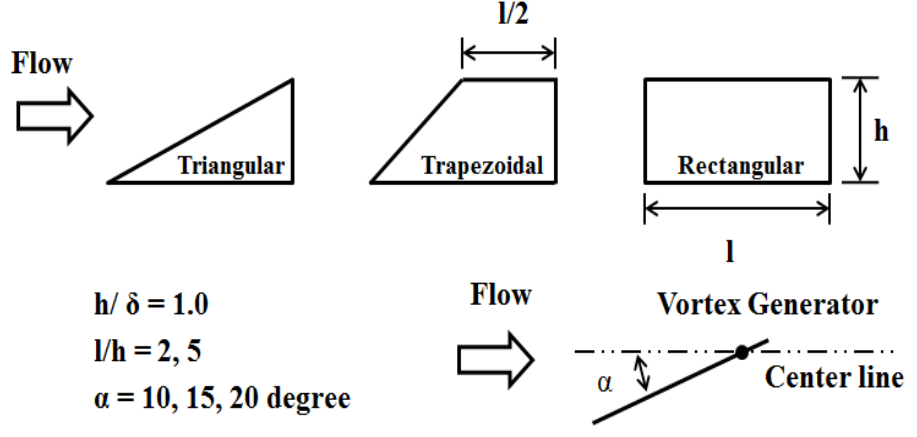

Fig. 2: Geometry of vortex generator and test conditions.

\subsection{Stereo-PIV}

Stereo-PIV was used for the wake measurements. Fig. 3 is a schematic of the experimental setup. As briefly illustrated in Fig. 3, two high resolution CCD cameras whose angle with respect to the laser light sheet was 45 degrees were placed at both sides of the test section at the same distance from the laser light sheet. A $200 \mathrm{~mJ}$ dual-head Nd:YAG laser of the QUANTEL Company was used. This provided the laser of $532 \mathrm{~nm}$ wavelength. The laser was illuminated vertically in the $\mathrm{x}-\mathrm{y}$ plane. As mentioned already, the surface of the flat plate was covered with black thin film not to allow the reflected laser into the CCD cameras.

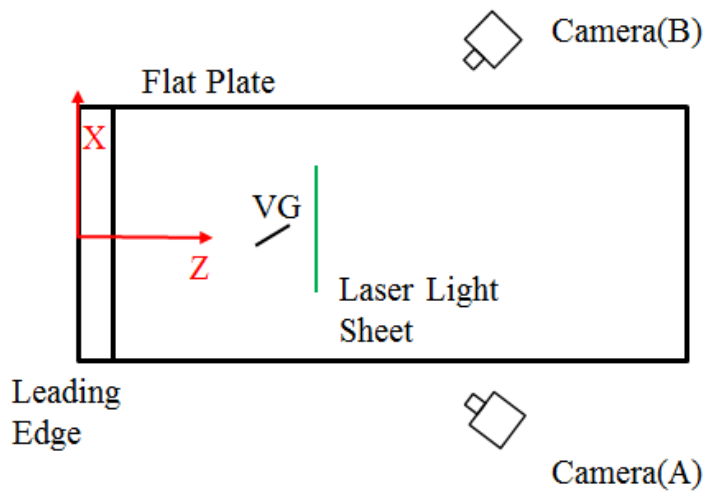

Fig. 3: Schematic of measurement system.

PIV images were acquired using the two high resolution $(2048 \times 2048$ resolutions $)$ cameras. The camera tilt angle was adjusted by the scheimpflug adapter which was used to reduce out of focus at the image corner. To supply the tracer particles, Laskin nozzle using DEHS-oil was installed at the test section breather, far downstream of the vortex generator. The average diameter of the particles was about $1 \mu \mathrm{m}$. The pulse generator of the BNC-555 model was used for 
synchronization between the laser and CCD cameras. The spatial resolution which affects the measurement result is defined by the interrogation window and overlap size. To estimate the effect of the spatial resolution, the image calculation was performed with respect to the various interrogation window and overlap size. First, the interrogation window size of $48 \times 48$ pixels was chosen through 2D displacement histogram analysis. Secondly, the overlap size was varied from $0 \%$ to $91 \%$, and $75 \%$ overlap size was selected. The $48 \times 48$ pixels and $75 \%$ overlap size implies that flow field have one vector for the area of $0.39 \mathrm{~mm} \times 0.39 \mathrm{~mm}$ for the laminar flow case. In the case of the turbulent flow, one vector spacing was $0.50 \mathrm{~mm} \times 0.50 \mathrm{~mm}$. Stereo-PIV measurements were performed at 7 downstream stations. Let $\Delta \mathrm{z}$ denote the distance of the measurement station from the trailing edge of the vortex generator. Yao et al. showed that the vorticity contours exhibited a fairly concentrated vortex structure within $\Delta \mathrm{z} / \mathrm{h} \leq 20$ [5]. In this study, 7 stations within $\Delta \mathrm{z} / \mathrm{h}<20$ were considered.

\section{Results}

\subsection{Mean Streamwise Flow Contour}

Fig. 4 shows mean streamwise velocity contours at one downstream station of the three vortex generators. The velocity contours are those viewed toward the upstream direction. The vortex generator projected onto each measurement station is also shown for convenience. As mentioned above, the heights of the vortex generator were approximately $5 \mathrm{~mm}$ and $15 \mathrm{~mm}$, respectively. The formation of the streamwise vortex is clearly seen in Fig. 4. The pressure side of the vortex generator corresponds to the positive coordinate side of Fig. 4. Thus, the rotational direction of the streamwise vortex is counter-clockwise as easily expected in these figures. Due to this vortex, the flow is swept upward in the pressure side region and downward in the suction side region resulting in greater streamwise mean velocity in the suction side. Interesting result in the laminar flow case was the generation of an additional vortex at specific test conditions. An additional vortex is observed at downstream of the vortex generator in Fig. 4. The additional vortex is referred to as secondary vortex. The secondary vortices seen in Fig. 4 are related to a horseshoe-like vortex around the leading edge of the vortex generator [10]. The rotation direction of the horseshoe vortex on the pressure side is in the same sign with the primary vortex. According to Velte et al., various regimes of the multi-vortical structure exist [10]. The flow condition, the geometric parameters of the vortex generator, and the interaction between the primary vortex and the horseshoe vortices affect these various regimes of the multi-vortical structure. Velte et al. mentioned three different regimes [10]. The vortical structure of the present of Fig. 4 complies with the first regime of Velte et al.

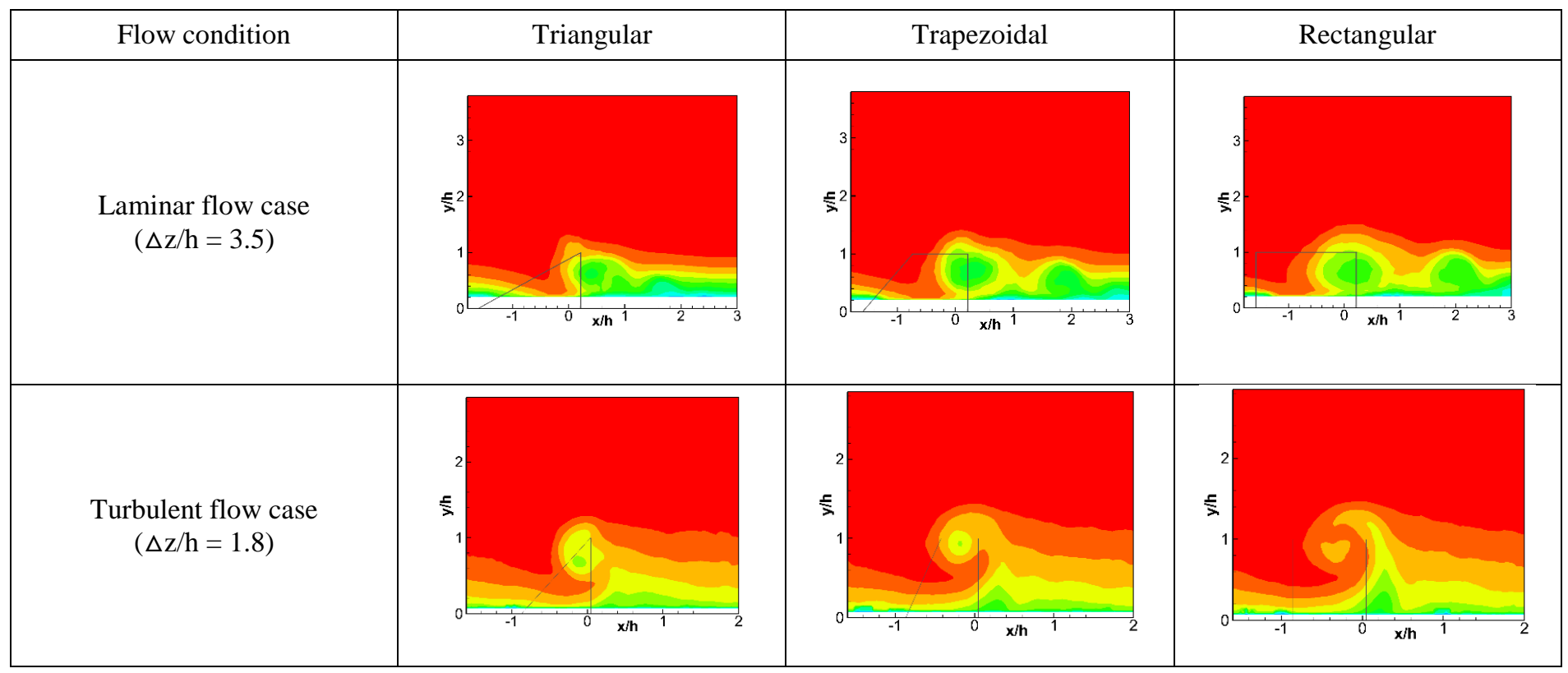

Fig. 4: Mean streamwise velocity contours for the laminar and the turbulent flow cases. 


\subsection{Streamwise Vorticity Distribution}

Table 1 lists the streamwise peak vorticity of the primary vortex of the three shapes of vortex generator at $\Delta \mathrm{z} / \mathrm{h}=1.5$ for the laminar flow case. For the case of the triangular generator, the peak vorticity is seen to increase with angle of attack at both length conditions. However, this tendency does not hold for the cases of trapezoidal and rectangular generator. Table 1 shows that the peak vorticity of the rectangular generator increases with angle of attack when $1 / \mathrm{h}=2$ but decreases with angle of attack when $1 / \mathrm{h}=5$. This reverse trend of the peak vorticity of the rectangular generator when $1 / \mathrm{h}=5$ will be discussed later. From Table 1, the peak vorticity variation of the trapezoidal generator with angle of attack is rather irregular and unusual compared to the other two generator cases. Table 2 lists the streamwise peak vorticities of the three shapes of vortex generator at $\Delta \mathrm{z} / \mathrm{h}=1.8$ for the turbulent flow case. When $1 / \mathrm{h}=2$, the rectangular generator at all angles of attack produces the largest peak vorticity. However, when $1 / \mathrm{h}=5$, this is not the case. At $\alpha=10^{\circ}$, the rectangular generator produces largest peak vorticity. At $\alpha=15^{\circ}$, the trapezoidal generator produces the largest peak whereas the triangular generator does at $\alpha=20^{\circ}$. For the rectangular and the trapezoidal generator cases, the peak vorticity at $\alpha=15^{\circ}$ is greater than that at $\alpha=20^{\circ}$. These are related to complicated separated flow structures around the vortex generators as commented in Yao et al. [5].

To examine the development properties of the streamwise vortex with downstream distance, variations of the peak vorticity with $\Delta \mathrm{z} / \mathrm{h}$ are depicted for the laminar flow case in Fig. 5. Here, $\Delta \mathrm{z}$ is the distance between the measuring station and the trailing edge of the vortex generator. The streamwise peak vorticities downstream of the generator were divided by the peak vorticity at $\Delta \mathrm{z} / \mathrm{h}=1.5$. Thus, at $\Delta \mathrm{z} / \mathrm{h}=1.5, \omega / \omega_{\max }=1.0$. Fig. 5 shows the variation of the streamwise peak vorticity of the primary vortex for the three vortex generator shapes of two different lengths at three angles of attack. Fig. 5 demonstrates evidently the effects of generator length and angle of attack. However, general tendency is not observed. Fig. 5 (f) shows that the decay rate of the triangular generator is much greater than that of the rectangular generator in the near wake region. From Fig. 5 (f) and Table $1\left(\alpha=20^{\circ}\right)$, even though the largest vorticity was generated by the triangular generator, the vorticity corresponding to the rectangular generator is seen to be much greater at further downstream stations $(\Delta \mathrm{z} / \mathrm{h}>5.5)$.

For the turbulent flow case, the streamwise peak vorticities at downstream were divided by the peak vorticity at $\Delta \mathrm{z} / \mathrm{h}$ $=1.8$. Thus, at $\Delta \mathrm{z} / \mathrm{h}=1.8, \omega / \omega_{\max }=1.0$. Fig. 6 demonstrate evidently the effects of generator length and angle of attack. Figs. 6 (a), 6 (b) illustrate that the vortex of the rectangular generator decays much faster in these cases, and Figs. 6 (e), 6 (f) show that the vortex of the triangular generator decays much faster in the near wake region. In the turbulent flow case, any general tendency can hardly be deduced.

Table 1: Peak vorticity of three shapes of vortex generator at $\Delta \mathrm{z} / \mathrm{h}=1.5$ for the laminar flow case.

\begin{tabular}{|c|c|c|c|c|}
\hline Length & $\alpha$ (deg.) & Triangular & Trapezoidal & Rectangular \\
\hline \multirow{3}{*}{$1 / h=2$} & 10 & 2697.5 & 4948.1 & 2786.0 \\
\cline { 2 - 5 } & 15 & 4565.1 & 5063.7 & 4219.5 \\
\cline { 2 - 5 } & 20 & 5196.6 & 4510.1 & 6722.2 \\
\hline \multirow{3}{*}{$1 / \mathrm{h}=5$} & 10 & 4202.7 & 6151.3 & 5226.8 \\
\cline { 2 - 5 } & 15 & 5474.9 & 5610.0 & 4645.0 \\
\cline { 2 - 5 } & 20 & 7100.7 & 5859.3 & 3924.5 \\
\hline
\end{tabular}

Table 2: Peak vorticity of three shapes of vortex generator at $\Delta \mathrm{z} / \mathrm{h}=1.8$ for the turbulent flow case.

\begin{tabular}{|c|c|c|c|c|}
\hline Length & $\alpha($ deg. $)$ & Triangular & Trapezoidal & Rectangular \\
\hline \multirow{3}{*}{$1 / \mathrm{h}=2$} & 10 & 2044.9 & 1929.4 & 5062.7 \\
\cline { 2 - 5 } & 15 & 2739.9 & 2496.3 & 5455.3 \\
\cline { 2 - 5 } & 20 & 2740.7 & 2356.0 & 2903.2 \\
\hline \multirow{3}{*}{$1 / \mathrm{h}=5$} & 10 & 2831.2 & 3330.1 & 4451.0 \\
\cline { 2 - 5 } & 15 & 4711.5 & 6195.5 & 5821.4 \\
\cline { 2 - 5 } & 20 & 7158.8 & 5754.1 & 5705.7 \\
\hline
\end{tabular}




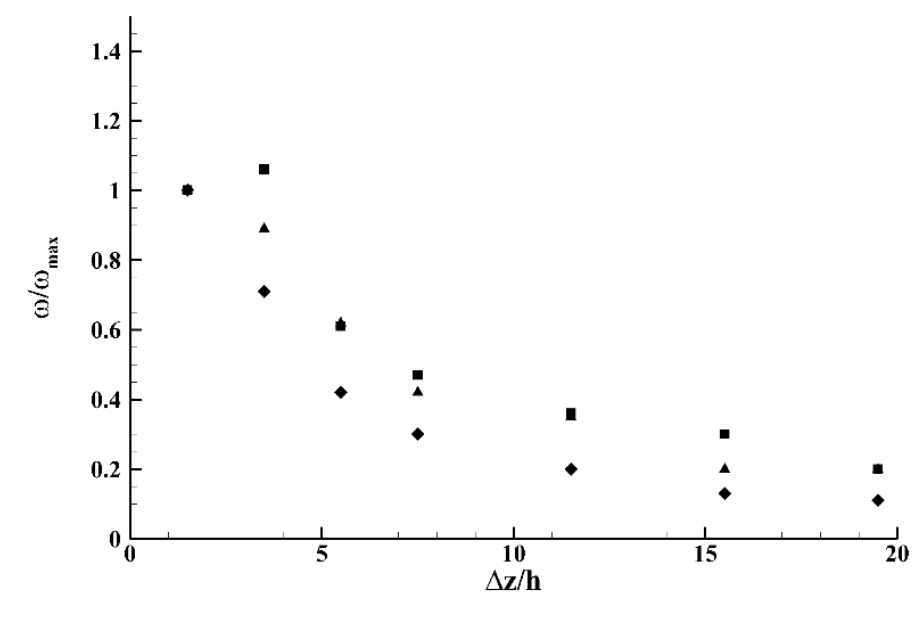

(a) $1 / \mathrm{h}=2$ and $\alpha=10 \mathrm{deg}$.

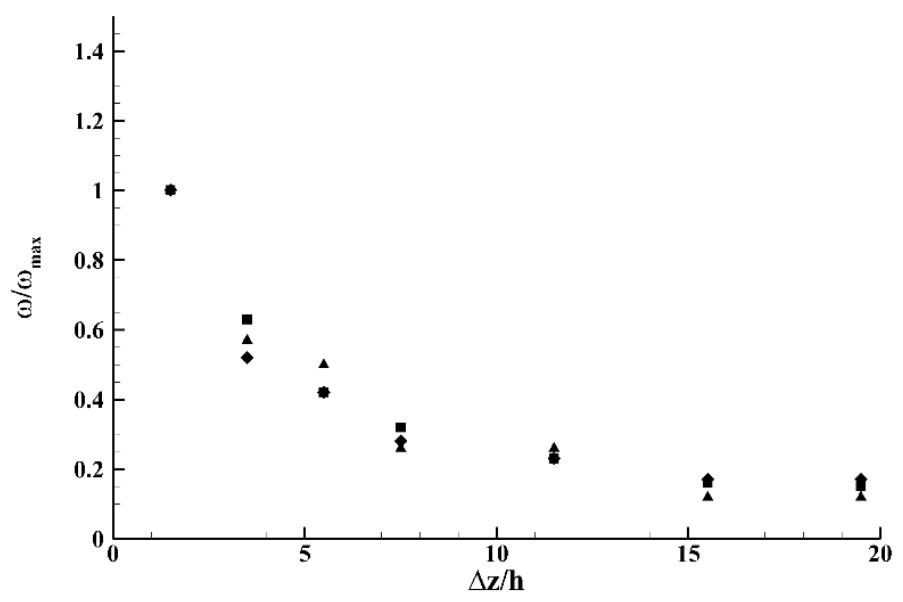

(b) $1 / \mathrm{h}=2$ and $\alpha=15 \mathrm{deg}$.

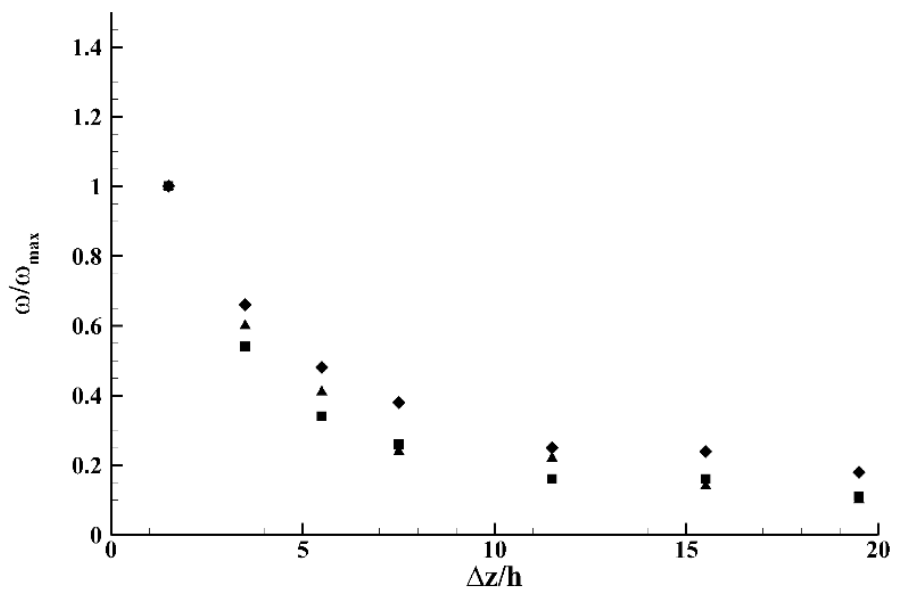

(c) $1 / \mathrm{h}=2$ and $\alpha=20 \mathrm{deg}$.

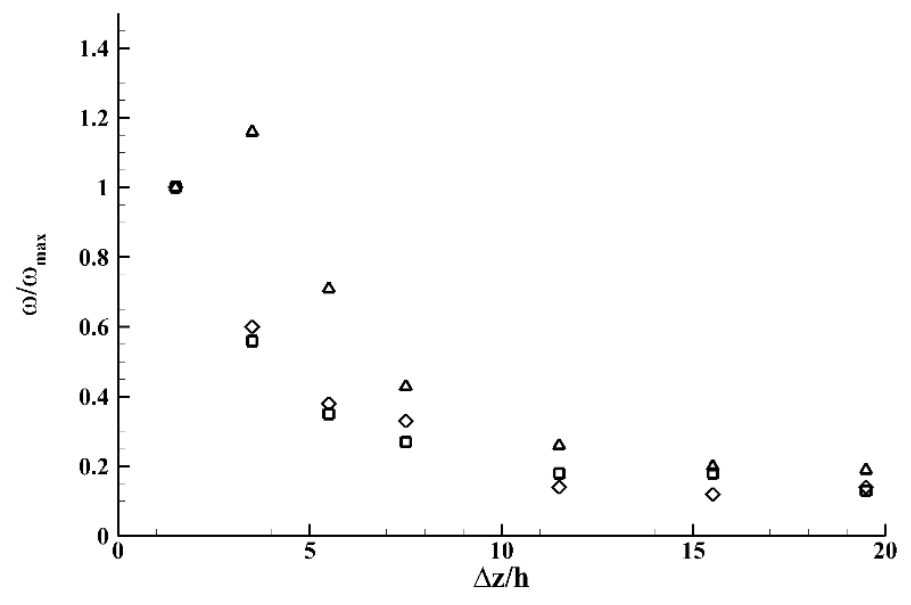

(d) $1 / \mathrm{h}=5$ and $\alpha=10 \mathrm{deg}$.

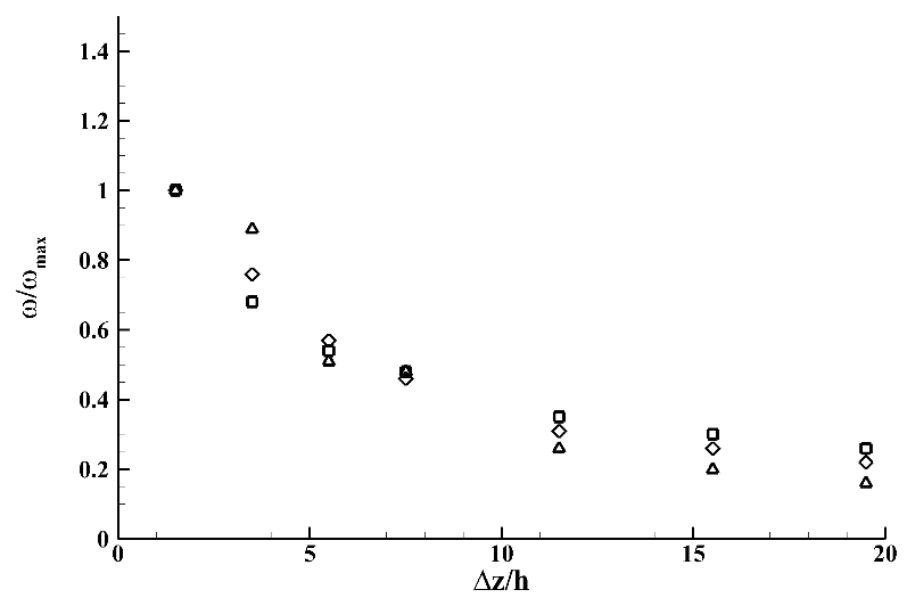

(e) $1 / \mathrm{h}=5$ and $\alpha=15 \mathrm{deg}$.

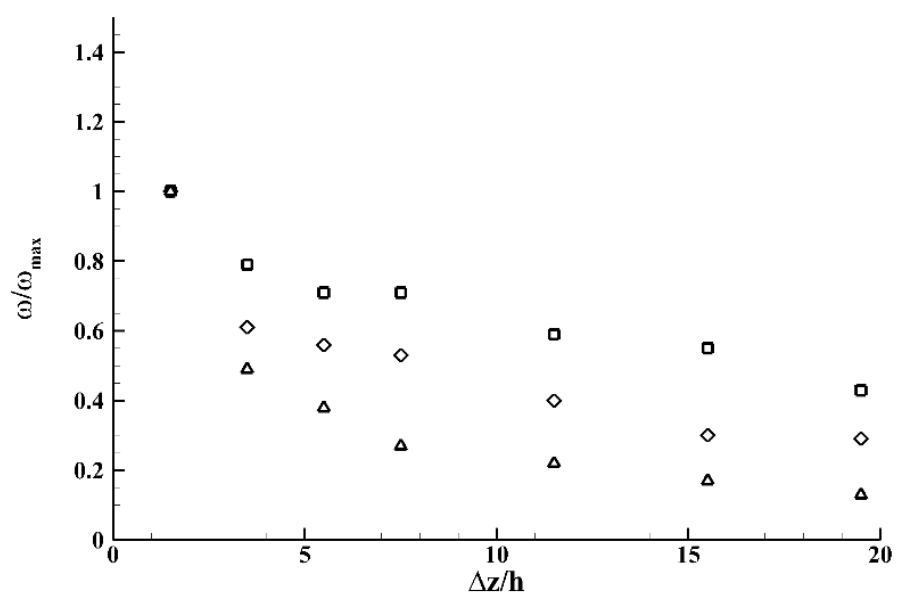

(f) $1 / \mathrm{h}=5$ and $\alpha=20 \mathrm{deg}$.

Fig. 5: Peak vorticity variations of primary vortex of three shapes of vortex generator for the laminar flow case ( $\boldsymbol{\Lambda}$ : triangular, : trapezoidal, and $\mathbf{\square}:$ rectangular generator when $1 / \mathrm{h}=2, \Delta:$ triangular, $\diamond:$ trapezoidal, and $\square:$ rectangular generator when $1 / \mathrm{h}=5$ ) 


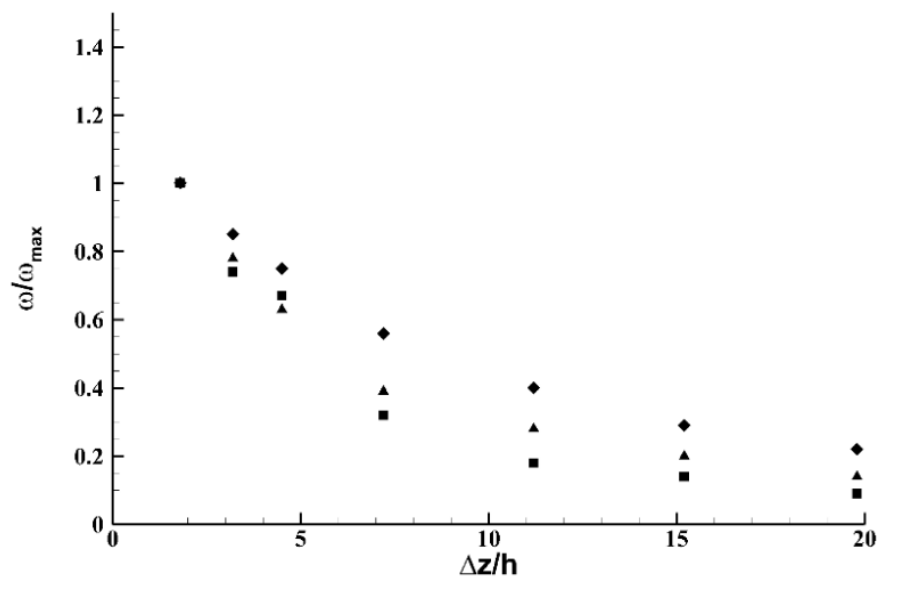

(a) $1 / \mathrm{h}=2$ and $\alpha=10 \mathrm{deg}$.

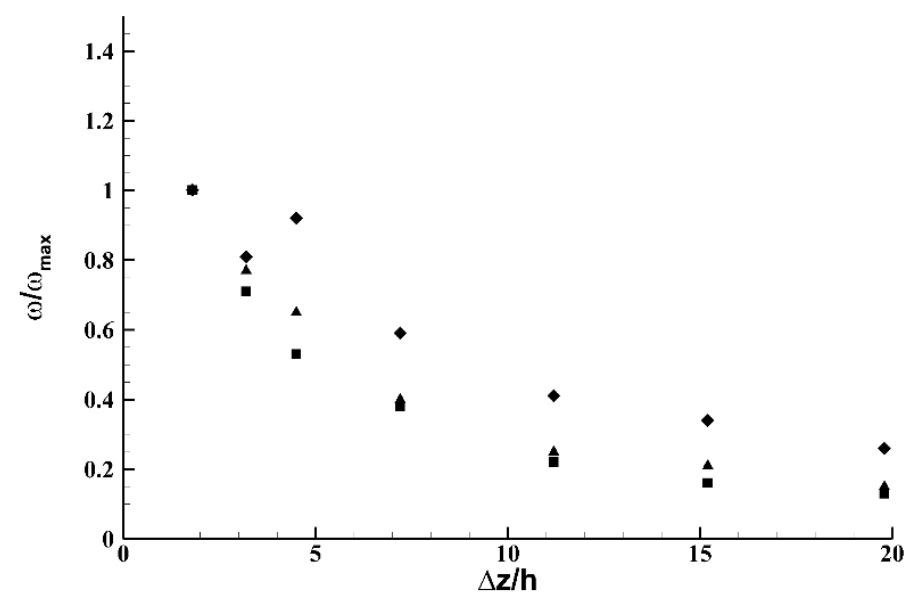

(b) $1 / \mathrm{h}=2$ and $\alpha=15 \mathrm{deg}$.

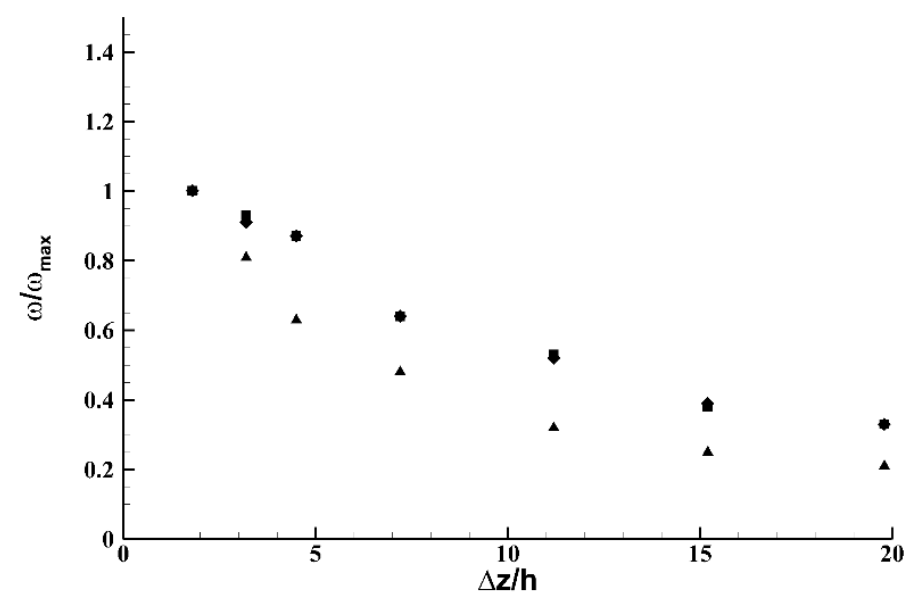

(c) $1 / \mathrm{h}=2$ and $\alpha=20 \mathrm{deg}$.

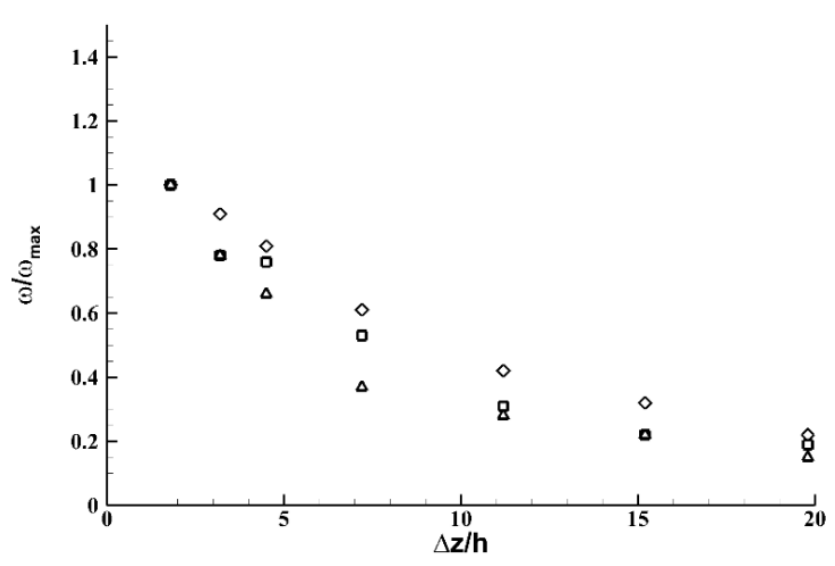

(d) $1 / \mathrm{h}=5$ and $\alpha=10 \mathrm{deg}$.

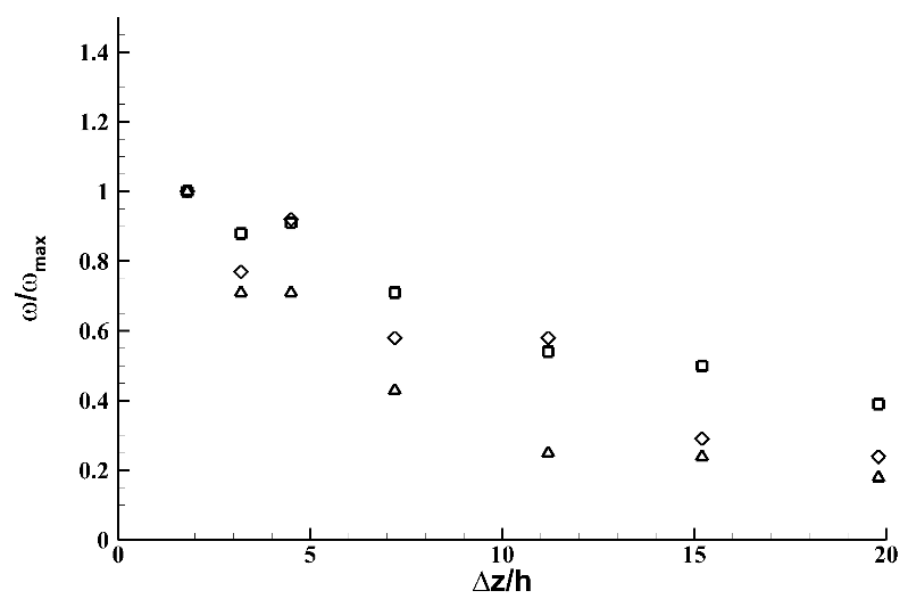

(e) $1 / \mathrm{h}=5$ and $\alpha=15 \mathrm{deg}$.

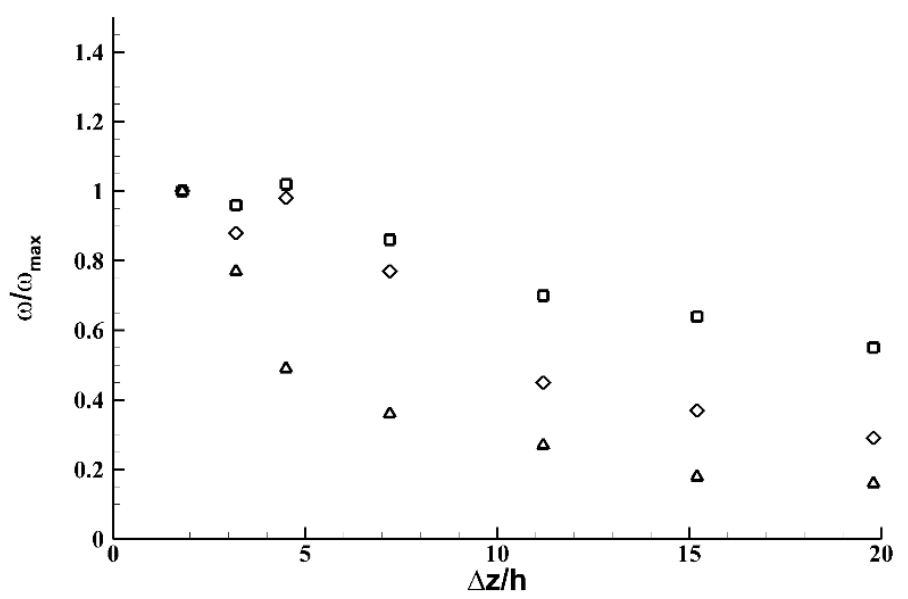

(f) $1 / \mathrm{h}=5$ and $\alpha=20 \mathrm{deg}$.

Fig. 6: Peak vorticity variations of primary vortex of three shapes of vortex generator for the turbulent flow case ( $\mathbf{\Lambda}$ : triangular, : trapezoidal, and $\mathbf{\square}$ : rectangular generator when $1 / \mathrm{h}=2, \Delta:$ triangular, $\diamond:$ trapezoidal, and $\square:$ rectangular generator when $1 / \mathrm{h}=5$ ). 


\section{Conclusion}

Experimental and investigations were conducted to elucidate the wake characteristics downstream of three vane-type vortex generators over a flat plate laminar and turbulent boundary layers. Experimental study was carried out by using stereoscopic particle image velocimetry. The triangular, the trapezoidal, and the rectangular generators of two different lengths at three angles of attack were tested.

In the case of the laminar flow case, two streamwise vortices at downstream of the generator were observed, the primary and secondary vortex, at some test cases illustrating that the flow structure is very complicated. The emergence of the secondary vortex depended on angle of attack and generator length. The decay rate of the primary vortex was evidently influenced by the generator length and angle of attack. When $\alpha=20^{\circ}$, for the case of $1 / \mathrm{h}=5$, even though the larger vorticity was generated by the triangular generator, the vorticity corresponding to the rectangular generator was greater at further downstream stations. This illustrates that the vorticity decay characteristics at high angle of attack with downstream distance also depend strongly on the generator shape. In the case of the turbulent flow case, the tendency of peak vorticity generation in the near wake region for each generator configuration was equal to the laminar flow case. However, in contrast with the laminar flow case, only a single streamwise vortex was observed for the turbulent flow case. For both cases of laminar and turbulent flow, the peak vorticity generation in the near wake region and the vorticity decay rates depends strongly on the generator shape and angle of attack. No general tendency was observed. This also signifies that the initial peak strength does not imply its strength tendency at further downstream stations.

\section{References}

[1] H. D. Taylor, "The Elimination of Diffuser Separation by Vortex Generators," United Aircraft Corporation Report No. R-4012-3.

[2] K. Torii, J. I. Yanagihara, "The Effects of Longitudinal Vortices on Heat Transfer of Laminar Boundary Layers," JSME, Series II, vil. 32, no. 3, pp. 395-402.

[3] J. I. Yanagihara, K. Torii, "Enhancement of Laminar Boundary Layer Heat Transfer by a Vortex Generator," JSME, Series II, vol. 35, no. 3, pp. 400-405.

[4] P. R. Ashill, J. L. Fulker, K. C. Hackett, "Research at DERA on Sub Boundary Layer Vortex Generators(SBVGs)," 39th Aerospace Sciences Meeting and Exhibit, AIAA 2001-0887.

[5] C. S. Yao, J. C. Lin, B. G. Allan, "Flow-Field Measurement of Device-Induced Embedded Streamwise Vortex on a Flat Plate," 1st AIAA Flow Control Conference, AIAA 2002-3162.

[6] K. P. Angele, B. Muhammad-Klingmann, "The effect of Streamwise Vortices on the Turbulence Structure of a Separating Boundary Layer," European Journal of Mechanics B/Fluids, 24, pp. 539-554.

[7] J. C. Lin, S. K. Robinson, R. J. McGhee, W. O. Valarezo, "Separation Control on High-Lift Airfoils via MicroVortex Generators," Journal of Aircraft, vol. 31, no. 6, pp. 1317-1323.

[8] G. Godard, M. Stanislas, "Control of a Decelerating Boundary Layer. Part I: Optimization of Passive Vortex Generators," Aerospace Science and Technology, 10, pp. 181-191.

[9] M. Kerho, S. Hutcherson, R. F. Blackwelder, R. H. Liebeck, "Vortex Generators used to Control Laminar Separation Bubbles," Journal of Aircraft, vol. 30, no. 3, pp. 315-319.

[10] C. M. Velte, V. L. Okulov, I. V. Naumov, "Regimes of Flow Past a Vortex Generator." Technical Physics Letter, vol. 38 , no. 4, pp. 379-382.

[11] J. I. Yanagihara, K. Torii, "Heat Transfer Augmentation by Longitudinal Vortices Rows," Experimental Heat Transfer, Fluid Mechanics and Thermodynamics, pp. 560-567.

[12] K. J. Kwon, "Experimental Study on Aerodynamic and Flow Field Characteristics of Elliptic Airfoils," Ph.D. dissertation, Korea Advanced Institute of Science and Technology, Daejeon, Republic of Korea.

[13] R. Narasimha, S. N. Prasad, "Leading Edge Shape for Flat Plate Boundary Layer Studies," Experiments in Fluids, vol. 17 , no. 5, pp. 358-360. 